\title{
Fibroepithelial lesions of the breast: a comprehensive morphological and outcome analysis of a large series
}

\author{
Elzbieta Slodkowska ${ }^{1,2} \cdot$ Sharon Nofech-Mozes ${ }^{1,2} \cdot$ Bin Xu (i) ${ }^{1,2} \cdot$ Carlos Parra-Herran $\mathbb{1}^{1,2} \cdot$ Fang-I Lu ${ }^{1,2} \cdot$ \\ Simon Raphael ${ }^{2,3,4} \cdot$ Judit Zubovits ${ }^{2,5} \cdot$ Wedad Hanna ${ }^{1,2}$
}

Received: 15 December 2017 / Revised: 16 January 2018 / Accepted: 16 January 2018 / Published online: 14 February 2018

(c) United States \& Canadian Academy of Pathology 2018

\begin{abstract}
Mammary fibroepithelial lesions encompass a wide spectrum of tumors ranging from an indolent fibroadenoma to potentially fatal malignant phyllodes tumor. The criteria used for their classification based on morphological assessment are often challenging to apply and there is no consensus as to what constitutes an adequate resection margin. We studied a retrospective cohort of 213 fibroepithelial lesions in 178 patients (80 fibroadenomas with unusual features and 133 phyllodes tumors: 63 benign, 41 borderline, and 29 malignant) in order to describe the spectrum of changes within each group, with special emphasis on margin evaluation. Outcome data were available for 153 fibroepithelial lesions in 139 patients (median 56 months, range 3-249 months). Positive final margin (tumor transected), age $<50$ years and a predominantly myxoid stroma were statistically significant predictors of local recurrence, while age $>50$, stromal overgrowth, diffuse marked atypia, necrosis and mitotic index of $\geq 10$ per $10 \mathrm{HPF}$ were predictive of distant metastases. Tumors with satellite/bulging nodules were at a significantly higher risk to have a final positive resection margin. Our findings highlight important aspects of the interpretation and reporting of fibroepithelial lesions: the amount of myxoid stroma and the presence of satellite nodules are clinically relevant and should be routinely assessed and reported; infiltrative border might not be a prerequisite for the diagnosis of malignant phyllodes tumor, while the presence of tumor necrosis, massive stromal overgrowth or mitotic index of $\geq 25$ per $10 \mathrm{HPF}$ is diagnostic of malignant phyllodes tumor. On the other hand, increased mitotic index outside of the range of the World Health Organization guidelines in the absence of other worrisome features should be treated with caution, as it can be found in benign tumors.
\end{abstract}

\section{Introduction}

Fibroepithelial lesions of the breast are biphasic neoplasms that comprise a wide spectrum of tumors ranging from the common indolent fibroadenoma to the rare malignant phyllodes tumor, with tumors of borderline clinical significance in between $[1,2]$. Classification of fibroepithelial

Elzbieta Slodkowska

elzbieta.slodkowska@sunnybrook.ca

1 Departments of Anatomic Pathology, Sunnybrook Health Sciences Centre, Toronto, ON, Canada

2 Department of Laboratory Medicine and Pathobiology, University of Toronto, Toronto, ON, Canada

3 North York General Hospital, Toronto, ON, Canada

4 Markham Stouffville Hospital, Markham, ON, Canada

5 Scarborough and Rouge Hospital, Scarborough, ON, Canada lesions relies purely on morphologic features, and while it has undergone consensus update in the recent past [2], it still poses an ongoing challenge to practicing pathologists. The current system of categorizing the morphologic continuum of the fibroepithelial lesions spectrum is based on multiple continuous variables and subjective cut offs ("mild" vs. "moderate" vs. "severe", "focal" as a cut off for "present"). This vaguely defined terminology creates substantial difficulty to classify lesions with overlapping features, even if combined with radiologic and clinical data. Moreover, the reproducibility of fibroepithelial lesions classification is suboptimal, especially at the benign end of the spectrum [3].

In the largest study of phyllodes tumors which included 605 cases, stromal atypia, mitoses, overgrowth, and surgical margins were independent significant predictors of recurrence free survival, with positive margins being the most important [4]. The challenges in assessing the clinical significance of these characteristics across studies are their inconsistent definitions and lack of a centralized pathology 
review process. Moreover, the validity of obtaining $1 \mathrm{~cm}$ clear margin for benign phyllodes tumors, which often results in suboptimal cosmetic outcome, has been recently challenged [5-7].

The purpose of this study was a detailed description of the frequency of morphologic findings within the spectrum of fibroepithelial lesions. Particular attention was paid to the margin status assessment, as well as to the low end of the spectrum (fibroadenoma vs. benign phyllodes tumor). A secondary goal was a search for features predictive of recurrence.

\section{Materials and methods}

We searched the Anatomic Pathology Laboratory Information System at Sunnybrook Health Sciences Centre (Toronto, Ontario, Canada) between 1994 and 2012 for patients diagnosed with phyllodes tumors, fibroepithelial lesions, and fibroadenomas with unusual features. The latter group included any of the following: cellular fibroadenoma, juvenile fibroadenoma, complex fibroadenoma, size at least $4 \mathrm{~cm}$ and diagnosed as fibroepithelial lesion favor fibroadenoma; for this study we referred to this group as atypical fibroadenoma. We only included cases with resection material available for pathology review. The original diagnosis, tumor size and mitotic index were extracted from pathology reports. All available slides were examined for the following characteristics (Fig. 1):

1. Tumor border: pushing (smooth vs. irregular/minimally infiltrative defined as microscopically irregular tumor interface with adjacent stroma (Fig. 1e)) vs. infiltrative (focally-unequivocal infiltration into adjacent breast stroma in 1-2 foci vs. widely-in $>2$ foci),

2. Satellite and/or bulging nodules (one or more tumor nodules separate from the main lesion or still connected and protruding from it into the surrounding tissue): absent vs. present,

3. Pseudocapsule (a rim of dense fibrous tissue at tumor border, complete or partial): absent vs. present,

4. Architecture: leafless vs. poorly developed or focal leaves vs. leafy (defined as having fibroepithelial fronds protruding into a cystically dilated space); lobular (defined as fibroepithelial lesion composed of lobules or nodules, either coalescing or separate),

5. Heterogeneity (differences in stromal quality and cellularity within fibroepithelial lesion): none vs. mild vs. moderate to marked,

6. Amount of myxoid stroma (as a percentage of the total surface area),

7. Stromal overgrowth (defined as $4 \times$ field without epithelium $\left(23.75 \mathrm{~mm}^{2}\right.$ field area): absent vs. present; in addition the number of $10 \times, 4 \times, 2 \times$ fields and entire slides (at least $2 \times 2 \mathrm{~cm}^{2}$-massive overgrowth) without epithelium was recorded,

8. Pseudoangiomatous stromal hyperplasia: absent vs. present (focal vs. diffuse),

9. Cellularity: \% moderate (stromal cells accounting for $30-80 \%$ of stromal surface), $\%$ marked (stromal cells closely packed accounting for $>80 \%$ of stromal surface),

10. Periductal hypercellularity (subepithelial stromal condensation): absent to inconspicuous vs. present (focal vs. diffuse); perivascular hypercellularity: absent vs. present,

11. Marked atypia: unequivocal atypia with enlarged pleomorphic hyperchromatic nuclei (defined as +++ by Moffat et al, reference 8); scattered (rare occasional cells) vs. focal vs. diffuse,

12. Maximal mitotic index: the maximal number of mitoses per a single set of 10 consecutive HPF $(40 \times$, $0.238 \mathrm{~mm}^{2}$ field area) in the most mitotically active area identified after a diligent review of all available slides,

13. Necrosis (defined as unequivocal geographic necrosis of phyllodes tumor stroma): absent vs. present (\%),

14. Infarction: absent vs. present (\%),

15. Malignant heterologous element: absent vs. present (type),

16. Lymphocytic infiltrate within fibroepithelial lesion: absent vs. minimal to mild (rare scattered stromal lymphocytes), vs. moderate (stromal lymphocytes accounting for up $10 \%$ of surface area) vs. marked ( $>10 \%$ ); presence of lymphoid aggregates within fibroepithelial lesions,

17. Epithelial changes within fibroepithelial lesion: usual ductal hyperplasia, adenosis, atypical ductal hyperplasia, lobular neoplasia (atypical lobular hyperplasia and/or lobular carcinoma in situ), ductal carcinoma in situ, invasive carcinoma,

18. Metaplasia within fibroepithelial lesion: epithelial (apocrine, squamous), stromal (myoid, osseous),

19. Fibroadenoma-like area(s) within phyllodes tumor (that could lead to a diagnosis of fibroadenoma on a core biopsy), hyalinized fibroadenoma within phyllodes tumor, absent vs. present,

20. Margin status: positive (fibroepithelial lesions transected) vs. negative (at least enucleated) vs. cannot assess (piecemeal removal, no ink); final margin was based on findings from the last surgery of the primary fibroepithelial lesion.

Based on this thorough morphological review fibroepithelial lesions were classified using the 2012 World Health Organization classification [1]. Lesions with some but not all the features from any given category were reviewed by at least two breast pathologist and a consensus diagnosis was reached. Fibroepithelial lesions that fell in between 2 categories were classified as that of a lower grade. Two cases of low-grade periductal stromal sarcoma 
Table 1 Summary of clinical and pathomorphological characteristics—categorical variables

\begin{tabular}{|c|c|c|c|c|c|c|c|c|c|}
\hline & $\begin{array}{l}\text { Atypical } \\
\text { fibro- } \\
\text { adenoma }\end{array}$ & & $\begin{array}{l}\text { Benign } \\
\text { phyllodes } \\
\text { tumor }\end{array}$ & & $\begin{array}{l}\text { Borderline } \\
\text { phyllodes } \\
\text { tumor }\end{array}$ & & $\begin{array}{l}\text { Malignant } \\
\text { phyllodes } \\
\text { tumor }\end{array}$ & & $P$ value \\
\hline & $N$ & $\%$ & $N$ & $\%$ & $N$ & $\%$ & $N$ & $\%$ & \\
\hline Total & 80 & 100 & 63 & 100 & 41 & 100 & 29 & 100 & 0.443 \\
\hline Age $<50$ y & 73 & 91 & 52 & 82 & 27 & 66 & 16 & 55 & $<0.001$ \\
\hline \multicolumn{10}{|l|}{ Laterality } \\
\hline $\mathrm{L}$ & 35 & 44 & 34 & 54 & 19 & 46 & 15 & 52 & 0.643 \\
\hline $\mathrm{R}$ & 45 & 56 & 29 & 46 & 22 & 54 & 14 & 48 & \\
\hline Size $>4 \mathrm{~cm}$ & 22 & 27 & 22 & 35 & 18 & 44 & 22 & 76 & $<0.001$ \\
\hline Final margin positive & 6 & 7 & 12 & 19 & 4 & 10 & 0 & 0 & 0.021 \\
\hline \multicolumn{10}{|l|}{ Infiltrative border } \\
\hline Any & $3^{\mathrm{a}}$ & 4 & $16^{\mathrm{a}}$ & 26 & 20 & 49 & 16 & 55 & $<0.001$ \\
\hline Widely infiltrative & 0 & 0 & 2 & 3 & 7 & 17 & 10 & 34 & \\
\hline Satellite/bulging nodules & 18 & 22 & 31 & 49 & 18 & 44 & 11 & 38 & 0.007 \\
\hline Pseudocapsule & 19 & 24 & 24 & 38 & 14 & 34 & 6 & 21 & 0.164 \\
\hline Lobular architecture & 54 & 67 & 42 & 67 & 27 & 66 & 14 & 48 & 0.284 \\
\hline \multicolumn{10}{|l|}{ Leafy architecture } \\
\hline Any & 25 & 31 & 50 & 79 & 37 & 91 & 18 & 62 & $<0.01$ \\
\hline Well developed & 2 & 2 & 18 & 29 & 18 & 44 & 12 & 41 & \\
\hline $\begin{array}{l}\text { Heterogeneity (at least } \\
\text { moderate) }\end{array}$ & 10 & 12 & 26 & 41 & 25 & 61 & 19 & 65 & $<0.001$ \\
\hline \multicolumn{10}{|l|}{ Myxoid stroma } \\
\hline$\geq 50 \%$ & 32 & 40 & 27 & 43 & 17 & 41 & 8 & 28 & 0.553 \\
\hline$\geq 90 \%$ & 11 & 14 & 14 & 22 & 6 & 15 & 5 & 17 & 0.575 \\
\hline $\begin{array}{l}\text { Pseudoangiomatous } \\
\text { stromal hyperplasia }\end{array}$ & 37 & 46 & 22 & 35 & 12 & 29 & 1 & 3 & $<<0.001$ \\
\hline \multicolumn{10}{|l|}{ Pure stroma in at least } \\
\hline $10 \times$ & 24 & 30 & 35 & 56 & 27 & 66 & 26 & 90 & $<0.001$ \\
\hline $4 \times$ & 0 & 0.0 & 4 & 6 & 9 & 22 & 26 & 90 & \\
\hline $2 \times$ & 0 & 0.0 & 0 & 0 & 0 & 0 & 20 & 70 & \\
\hline Entire slide & 0 & 0.0 & 0 & 0 & 0 & 0 & 16 & 55 & \\
\hline \multicolumn{10}{|l|}{ Periductal hypercellularity } \\
\hline Any & 51 & 64 & 49 & 78 & 37 & 90 & 13 & 45 & $<0.001$ \\
\hline Diffuse & 3 & 4 & 16 & 25 & 25 & 61 & 8 & 28 & \\
\hline $\begin{array}{l}\text { Perivascular } \\
\text { hypercellularity }\end{array}$ & 1 & 1 & 3 & 5 & 2 & 5 & 5 & 17 & $<0.001$ \\
\hline \multicolumn{10}{|l|}{ Marked nuclear atypia } \\
\hline Any & 2 & 2 & 6 & 9 & 20 & 49 & 23 & 79 & $<0.001$ \\
\hline Diffuse & 0 & 0 & 0 & 0 & 10 & 24 & 21 & 72 & \\
\hline Necrosis & 0 & 0 & 0 & 0 & 0 & 0 & 12 & 41 & $<0.001$ \\
\hline Infarction & 1 & 1 & 5 & 8 & 7 & 17 & 2 & 7 & 0.015 \\
\hline $\begin{array}{l}\text { Heterologous malignant } \\
\text { element }\end{array}$ & 0 & 0 & 0 & 0 & 0 & 0 & 4 & 14 & $<0.001$ \\
\hline Lymphoid infiltrate & 34 & 42 & 28 & 44 & 24 & 58 & 10 & 34 & 0.209 \\
\hline Lymphoid aggregates & 30 & 37 & 35 & 56 & 20 & 49 & 12 & 41 & 0.171 \\
\hline Usual ductal hyperplasia & 14 & 17 & 28 & 44 & 21 & 51 & 6 & 21 & $<0.001$ \\
\hline Adenosis & 18 & 22 & 10 & 16 & 8 & 19 & 3 & 10 & 0.483 \\
\hline
\end{tabular}


Table 1 (continued)

\begin{tabular}{|c|c|c|c|c|c|c|c|c|c|}
\hline & $\begin{array}{l}\text { Atypical } \\
\text { fibro- } \\
\text { adenoma }\end{array}$ & & $\begin{array}{l}\text { Benign } \\
\text { phyllodes } \\
\text { tumor }\end{array}$ & & $\begin{array}{l}\text { Borderline } \\
\text { phyllodes } \\
\text { tumor }\end{array}$ & & $\begin{array}{l}\text { Malignant } \\
\text { phyllodes } \\
\text { tumor }\end{array}$ & & $P$ value \\
\hline & $N$ & $\%$ & $N$ & $\%$ & $N$ & $\%$ & $N$ & $\%$ & \\
\hline Apocrine metaplasia & 18 & 22 & 14 & 22 & 10 & 24 & 2 & 7 & 0.266 \\
\hline $\begin{array}{l}\text { Fibroadenoma-like area in } \\
\text { phyllodes }\end{array}$ & $\mathrm{n} / \mathrm{a}$ & $\mathrm{n} / \mathrm{a}$ & 55 & 87 & 28 & 68 & 14 & 48 & 0.335 \\
\hline Hyalinized fibroadenoma & $\mathrm{n} / \mathrm{a}$ & $\mathrm{n} / \mathrm{a}$ & 21 & 33 & 14 & 34 & 8 & 28 & 0.136 \\
\hline
\end{tabular}

${ }^{a}$ Border unknown in one atypical fibroadenoma and one benign phyllodes tumor due to piecemeal removal

were classified as benign phyllodes tumors for statistical purposes. Follow-up information, mode of treatment and previous history of fibroadenoma other than the index lesion were obtained from electronic medical records; in 6 patients the cause of death was confirmed through Cancer Care Ontario.

All statistical analyses were performed using the SPSS software 24.0 (IBM Corporation, New York, NY, US). Clinico-pathological characteristics were compared between different types of fibroepithelial lesions using appropriate statistical tests, i.e., Chi-square test or Fisher's exact test for nonparametric variables and one-way analysis of variance (ANOVA) for continuous variables. Recurrence free survival was calculated from the date of diagnosis to the date of recurrence. Prognostic value of each clinico-pathological feature was determined using univariate Log rank test and recurrence free survival. For local recurrence the pathological features in all fibroepithelial lesions were analyzed, while for distant recurrence analysis only phyllodes tumors were included. Prognostic variables that were significant on univariate analyses were subsequently subjected to multivariate analyses using the Cox proportional hazards model. $P$ values $<0.05$ were considered to be statistically significant.

The study was approved by Research Ethics Boards of the involved institutions.

\section{Results}

\section{Characteristics of the study cohort}

213 fibroepithelial lesions from 178 patients that fulfilled the inclusion criteria described above were included in the present study and classified as follows: 80 atypical fibroadenomas in 68 patients (3 also had benign phyllodes tumors), 63 benign phyllodes tumors in 53 patients ( 8 of them were recurrences), 41 borderline phyllodes tumors in 38 patients (5 represented recurrences) and 29 malignant phyllodes tumors in 29 patients ( 7 were recurrent tumors). Twelve patients had multiple (ranging from 2 to 4 ) index fibroepithelial lesions; 7 patients had bilateral index fibroepithelial lesions, either atypical fibroadenoma or benign phyllodes tumors or combination of the two.

In 63 cases fibroepithelial lesions were reclassified as compared to the original diagnosis-48 were downgraded (the vast majority moved from benign phyllodes tumor to atypical fibroadenoma), 15 upgraded and 10 changed from fibroepithelial lesion/phyllodes tumor to a specific category.

Only 18 fibroepithelial lesions (8\%) included in this study were diagnosed prior to 2000 and $173(81 \%)$ after 2004. The majority of the cases $(77 \%)$ had their surgery and primary pathology assessment at Sunnybrook Hospital and $49(23 \%)$ of the cases were referred in from 3 hospitals in the Greater Toronto Area (including 18 atypical fibroadenomas, 15 benign, 15 borderline, and 1 malignant phyllodes tumor). On average one slide per $1 \mathrm{~cm}$ of fibroepithelial lesion was available for microscopic examination.

\section{Morphological analysis}

The morphological characteristics of the cohort are summarized in Tables 1 and 2, and are further described in the following paragraphs.

\section{Tumor border}

Infiltrative tumor border was observed across the spectrum of fibroepithelial lesions with significantly different frequency and extent $(p<0.01)$. The vast majority $(77 / 80$, $96 \%$ ) of atypical fibroadenomas did not exhibit any evidence of infiltrative border with the exception of three cases showing focal infiltration (Fig. 1d). In benign phyllodes tumors infiltrative border, if present, was usually focal (14/ $16,87 \%$ of benign phyllodes with infiltrative border). In contrast, widely infiltrative border was seen with increasing frequency in borderline $(7 / 41,17 \%)$ and malignant phyllodes tumors $(10 / 29,35 \%)$. 


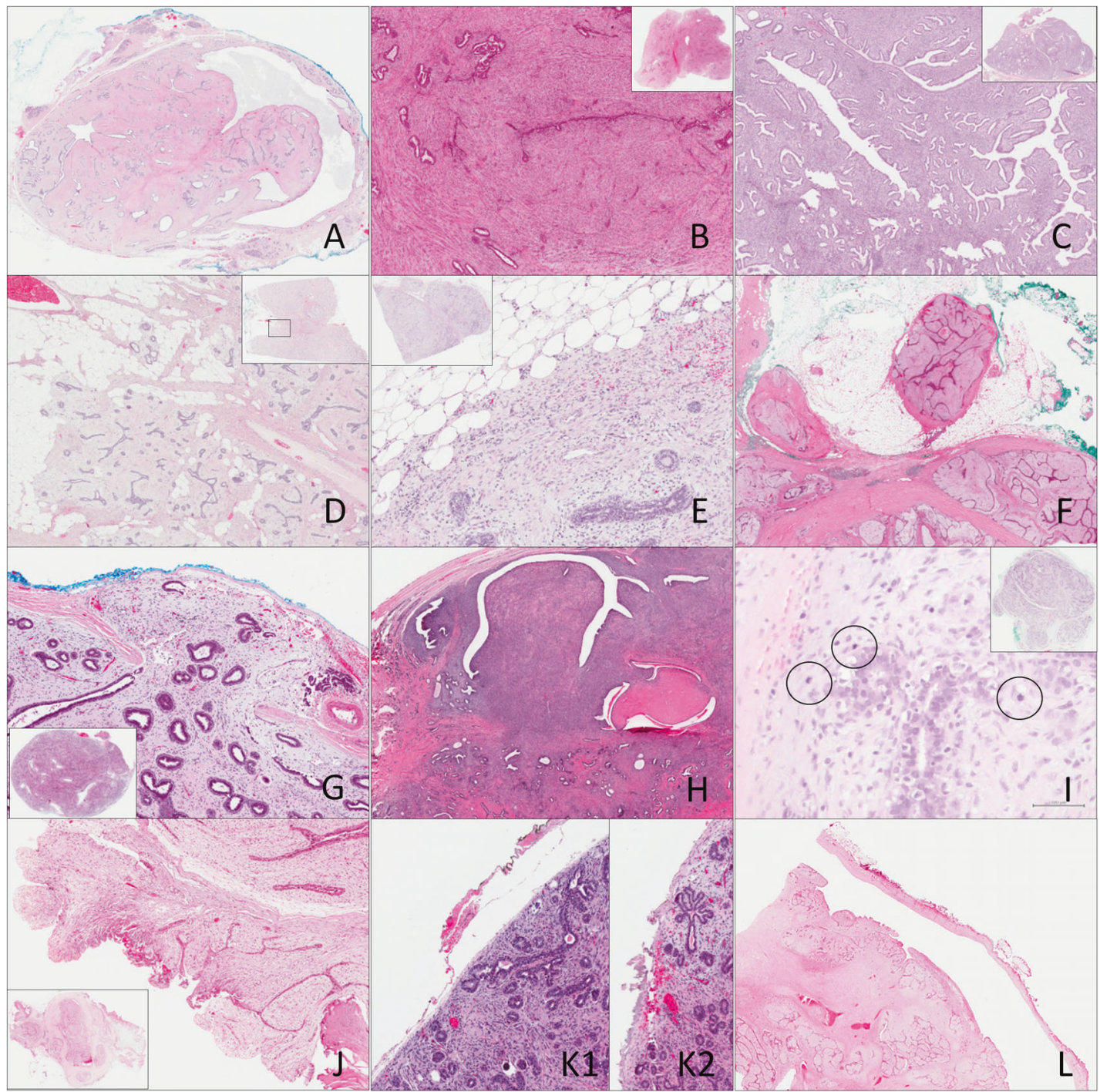

Fig. 1 a Leafy fibroadenoma, b leafless phyllodes, c malignant phyllodes tumor with abundant epithelium and therefore no stromal overgrowth, d fibroadenoma with focally infiltrative margin, e irregular/minimally infiltrative margin, $\mathbf{f}$ satellite nodules and partial pseudocapsule, $\mathbf{g}$ bulging through pseudocapsule, $\mathbf{h}$ leaf and marked heterogeneity, i variant fibroadenoma with high mitotic index (2 mitoses in a single HPF), $\mathbf{j}$ positive margin (transected), $\mathbf{k}$ false positive margin ( $\mathrm{K} 1$-thin film of compressed normal tissue around fibroadenoma, K2-cautery on tumor as this thin film was damaged during surgery), I separation of pseudocapsule/thin rim of compressed uninvolved tissue that may contribute to false positive margin. Inset (if present) represents whole slide scan
Satellite and/or bulging nodules were seen in a minority of fibroepithelial lesions across the spectrum. The frequency differed across the categories and was significantly higher in phyllodes tumors $(p=0.007$, Table 1).

\section{Architecture and heterogeneity}

Well developed, diffuse leafy architecture was present in $29 \%$ (18/63) of benign, $44 \%$ (18/41) of borderline and $41 \%$ $(12 / 29)$ of malignant phyllodes tumors, as well as in $2 \%(2 /$ $80)$ of atypical fibroadenomas $(p<0.001) ; 21 \%(13 / 63)$ of benign, $10 \%(4 / 41)$ of borderline and $38 \%(11 / 29)$ of malignant phyllodes tumors were leafless. In malignant phyllodes the leafy architecture was either non-questionable or completely absent (in cases with massive stromal overgrowth and only minimal epithelial component). Heterogeneity was most pronounced in the malignant end of the spectrum-61\% (25/41) of borderline and 65\% (19/29) of malignant phyllodes tumors displayed at least moderate heterogeneity.

\section{Stromal cellularity}

Increased cellularity was seen in all four groups; as many as $29 \%$ (23/80) atypical fibroadenomas and 29\% (18/63) benign phyllodes tumors had predominantly moderate 


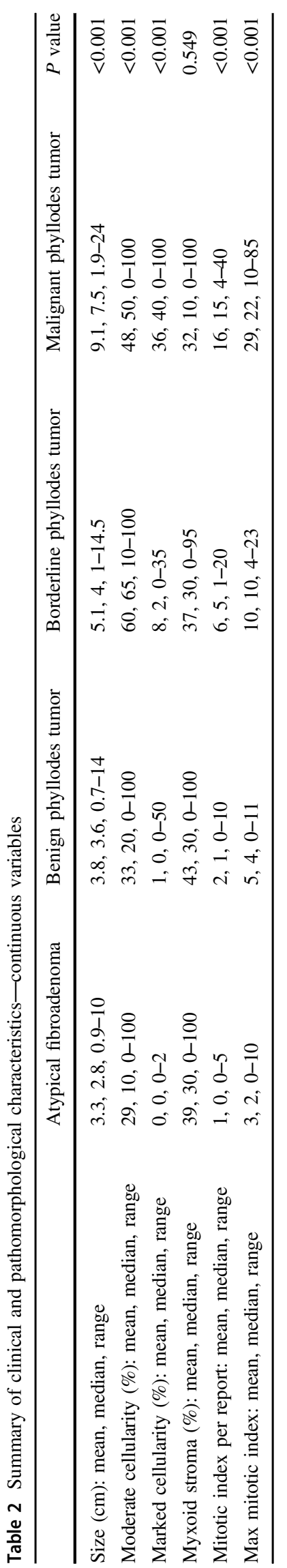

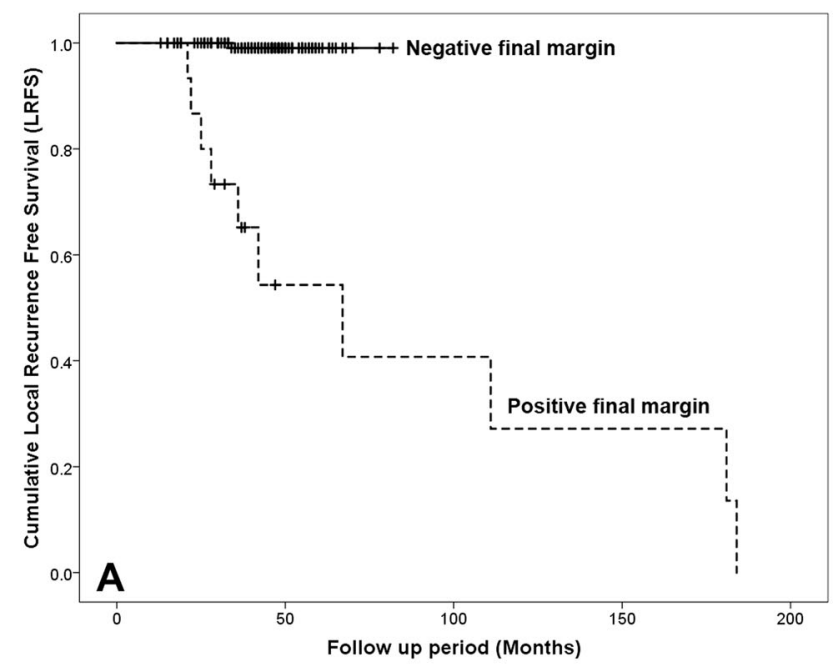
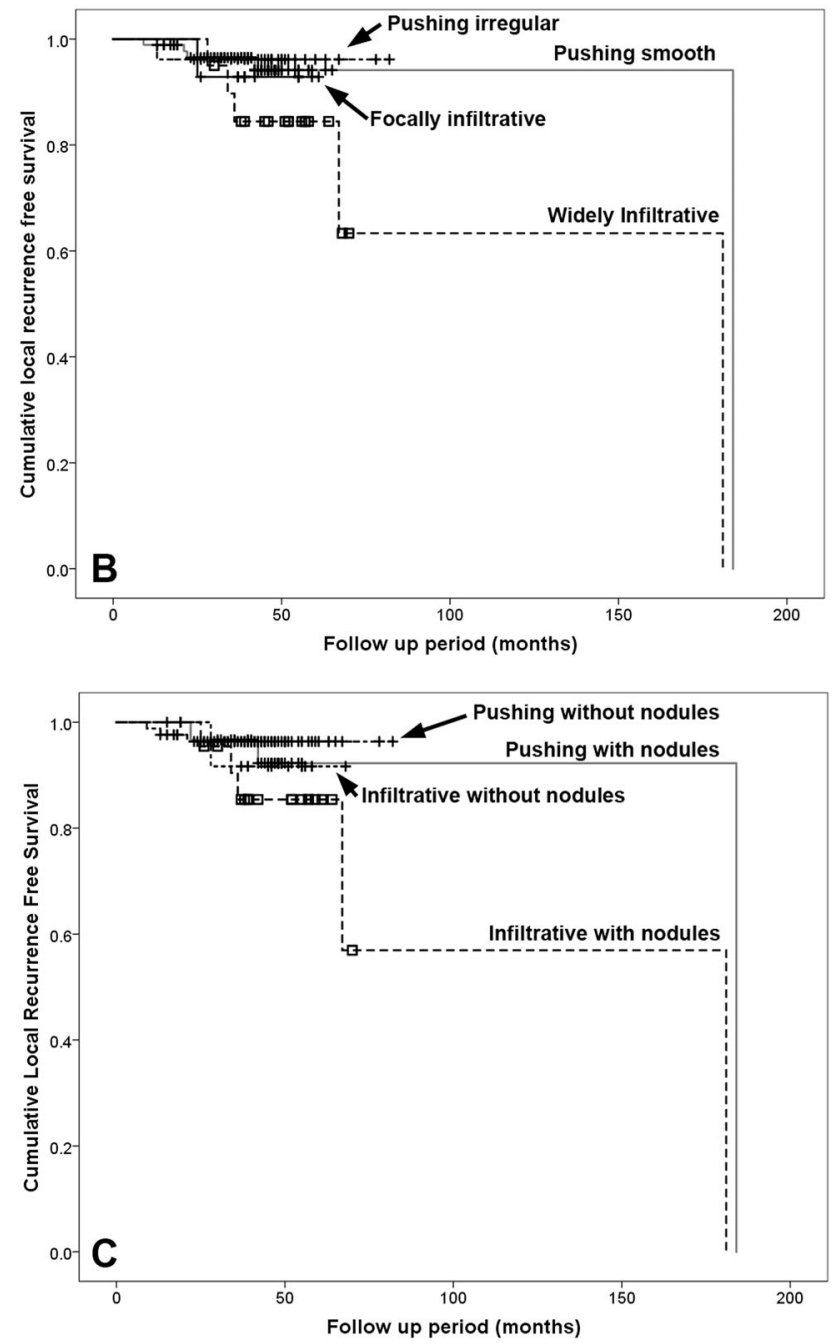

cellularity while moderate to marked cellularity in $>50 \%$ of stroma was seen in 78\% (32/41) borderline and all 29 malignant phyllodes tumors. Marked cellularity on the other hand was extremely rare and usually very limited in amount 
Fig. 2 a Positive final margin was a significant predictor of local recurrence free survival. b For local recurrence free survival there was a near significant difference between fibroepithelial lesions with widely infiltrative border (group 4) and the remaining three groups (pushing, minimally infiltrative and focally infiltrative, $p=0.079$ ). c When comparing fibroepithelial lesions in relation to their border including the presence of satellite/bulging nodules there was a near significant difference for local recurrence free survival between pushing border without nodules and infiltrative border with nodules ( $p$ $=0.061)$

in the benign end of the spectrum (present in two atypical fibroadenomas accounting for 1-2\% of stroma and in $14 \%$ (9/63) benign phyllodes tumors, in 7 of them only accounting for $<5 \%$ of stroma), while it was present in most borderline $(51 \%, 21 / 41)$ and malignant phyllodes tumors (90\%, 26/29). The two cases of benign phyllodes tumors with marked cellularity of $25 \%$ and $50 \%$, respectively, were morphologically unusual; despite marked stromal cellularity the mitoses were sparse ( $<5$ per $10 \mathrm{HPF}$ ), there was no stromal overgrowth or atypia and the border was pushing; therefore they were not assigned to a higher category. Periductal hypercellularity was commonly seen as a focal feature; in diffuse form it was very rare in atypical fibroadenomas $(3 / 80,4 \%)$ and most common in borderline phyllodes tumors $(25 / 41,61 \%)$.

\section{Stromal characteristics}

Myxoid stroma was common throughout the spectrum of fibroepithelial lesions with the average range from 32 to $43 \%(p=0.549)$; also lesions with predominantly myxoid stroma were seen in fairly equal distribution along the entire spectrum $(p=0.500)$. Pseudoangiomatous stromal hyperplasia was commonly present in the benign end of the spectrum and was only focally seen in one malignant phyllodes $(p<0.001)$. Stromal overgrowth was rare outside of malignant phyllodes tumors (absent in fibroadenomas, present in $6 \%$ (4/63) of benign, $22 \%$ (9/41) of borderline and $90 \%(26 / 29)$ of malignant phyllodes. However, at least one $10 \times$ field with pure stoma was present in $30 \%(24 / 80)$ of atypical fibroadenomas, 56\% (35/63) of benign, 66\% (27/ 41 ) of borderline and $90 \%$ (26/29) malignant phyllodes tumors. Three malignant phyllodes had abundant epithelial component with not a single $10 \times \mathrm{HPF}$ of pure stroma (all 3 had tumor necrosis, moderate to marked cellularity in at least $70 \%$ of stroma, marked atypia, and mitotic index of at least 25 per HPF; one had malignant heterologous element). Only malignant phyllodes tumors had necrosis and/or malignant heterologous element (6 cases overall, liposarcoma, chondrosarcoma and osteosarcoma alone or in combination in 2 cases each). Areas resembling usual fibroadenoma were seen in the vast majority of benign and borderline phyllodes tumors ( 87 and $68 \%$, respectively) and in $48 \%$ of malignant phyllodes tumors. A hyalinized fibroadenoma within phyllodes tumor was seen in $28-34 \%$ cases across the spectrum. Myoid metaplasia was present in 6 atypical fibroadenomas ( $7 \%), 3$ benign phyllodes tumors $(5 \%)$ and none of the tumors from the malignant end of the spectrum, while osseous metaplasia was seen in only one case (borderline tumor). Infarction was rarely seen in all 4 groups (1 atypical fibroadenoma involving $60 \%$ of volume, 5 benign phyllodes tumors $(8 \%)$ from $5-80 \%$ of tumor volume, 7 borderline phyllodes tumors (17\%) from $<5-40 \%$ of tumor volume and 2 malignant phyllodes tumors $(7 \%)$ involving $10 \%$ of tumor volume in each).

\section{Atypia and mitotic index}

Marked stromal cytologic atypia was not present in the benign end of the spectrum unless as scattered single cells (seen in 3 cases of benign phyllodes tumors and not seen in fibroadenomas). In terms of mitotic index rare atypical fibroadenoma had up to 10 mitoses per $10 \mathrm{HPF}$ in the most mitotically active area (2/80 cases with 10 mitoses per 10 HPF; 5/80 cases with $>5$ mitoses per $10 \mathrm{HPF}$ ), while one borderline tumor had $<5$ mitoses per $10 \mathrm{HPF}$ (together with moderate to marked cellularity and diffuse marked stromal cell atypia). Five (8\%) benign phyllodes tumors had 10-13 mitoses, while $24(58 \%)$ borderline tumors had 10 or more mitoses per $10 \mathrm{HPF}$. All malignant tumors had at least 10 mitoses per $10 \mathrm{HPF}$ after a diligent search; only malignant phyllodes tumors had 25 or more mitoses per $10 \mathrm{HPF}$.

\section{Epithelial component}

Metaplastic changes were present in about one third of fibroadenomas, benign and borderline phyllodes tumors each, and were rare in malignant ones (10\%). Invasive mammary carcinoma was seen in 2 cases (both benign phyllodes tumors), ductal carcinoma in situ in none, and atypical ductal hyperplasia in rare fibroadenomas and benign phyllodes; lobular neoplasia was present in 8 cases overall (mostly in borderline phyllodes tumors).

Apocrine metaplasia was present in up to almost a quarter of all lesions from each group except for malignant phyllodes tumors $(7 \%)$; squamous metaplasia was seen in only 4 cases overall.

\section{Lymphocytic infiltrate}

Prominent lymphocytic infiltrate was rare throughout the spectrum ( 5 atypical fibroadenomas, 8 benign, 4 borderline, and 3 malignant phyllodes tumors). Lymphoid aggregates, usually very few, were seen in 30 (37\%) fibroadenomas, 37 (59\%) benign, 20 (49\%) borderline, and 12 (41\%) malignant phyllodes tumors. 
Table 3 Summary of treatment, follow-up, and recurrences

\begin{tabular}{|c|c|c|c|c|}
\hline & Atypical fibroadenoma & Benign phyllodes tumor & $\begin{array}{l}\text { Borderline phyllodes } \\
\text { tumors }\end{array}$ & $\begin{array}{l}\text { Malignant phyllodes } \\
\text { tumors }\end{array}$ \\
\hline Mastectomy as primary treatment & $1(1 \%)$ & $1(2 \%)$ & $3(7 \%)$ & $8(28 \%)$ \\
\hline Re-excision ${ }^{\mathrm{a}}$ : any, mastectomy & $6(8 \%), 1(1 \%)$ & $12(25 \%), 2(4 \%)$ & $18(62 \%), 3(10 \%)$ & $13(81 \%), 9(56 \%)$ \\
\hline Adjuvant therapy & $\mathrm{n} / \mathrm{a}$ & $1(2 \%)$ & $4(12 \%)$ & $7(32 \%)$ \\
\hline Patients with follow-up & 51 & 43 & 28 & 21 \\
\hline $\begin{array}{l}\text { Fibroepithelial lesions with follow- } \\
\text { up }\end{array}$ & 59 & 45 & 28 & 21 \\
\hline Follow-up: median, range (months) & $56,3-210$ & $60,3-194$ & $48,6-249$ & $49,8-207$ \\
\hline Outcome per fibroepithelial lesion & NED 59 & $\begin{array}{l}\text { NED } 43 \text { AWD } 1 \text { DOD } \\
1^{\mathrm{b}}\end{array}$ & NED 28 & 13 NED 7 DOD 1 DOC \\
\hline Lymph node metastases & 0 & 0 & 0 & $2^{\mathrm{c}}$ \\
\hline Local recurrence $^{\mathrm{d}}$ & 0 & $14(31 \%)$ & $4(14 \%)$ & $1(5 \%)$ \\
\hline Distant metastases & 0 & 0 & 0 & $7(33 \%)$ \\
\hline
\end{tabular}

$N E D$ no evidence of disease, $A W D$ alive with disease, $D O D$ dead of disease, $D O C$ dead of other causes

${ }^{a}$ Cases with mastectomy as primary treatment excluded from calculation

${ }^{\mathrm{b}}$ Recurred as malignant phyllodes

${ }^{\mathrm{c}}$ Axillary node sampling performed in five patients

${ }^{\mathrm{d}}$ For additional two recurrent malignant phyllodes the original tumor was unknown

\section{Margin status}

The final resection margin was negative in 74 (92\%) atypical fibroadenomas, $45(82 \%)$ benign, 33 (92\%) borderline and all 22 malignant phyllodes tumors; 54 (67\%) fibroadenomas, $17(31 \%)$ benign, $12(33 \%)$ borderline and 5 (23\%) malignant phyllodes tumors were enucleated (shelled out) at the final surgical procedure. For the 153 fibroepithelial lesions with known margin and follow-up 10 of 15 with positive (transected) margin recurred; the remaining 5 were either atypical fibroadenomas or benign phyllodes tumors with only focally positive margin.

\section{Treatment, follow-up, and recurrence rate}

Treatment is summarized in Table 3. Re-excision for positive or close margins was performed in $12(25 \%)$ benign, 18 $(62 \%)$ borderline and $13(81 \%)$ malignant phyllodes tumors; no residual tumor was found in any of the reexcision specimens. Adjuvant radiotherapy was given to 7 (32\%) patients with malignant phyllodes tumor, 4 (12\%) patients with primary borderline and 1 patient with benign phyllodes tumor (originally diagnosed as borderline, reclassified as benign phyllodes); one patient with malignant tumor also received adjuvant chemotherapy. Radiotherapy was also given to 2 and 3 patients with recurrent borderline and malignant phyllodes tumors, respectively. Chemotherapy was given to 4 patients with metastatic tumors and one with concurrent invasive mammary carcinoma.
Follow-up was available for 153 fibroepithelial lesions in 139 patients with a median of 56 months (mean: 67 months, range: 3-249 months). Seven patients (5\%) died of disease (all with malignant phyllodes). One died of breast cancer having concurrent ipsilateral malignant phyllodes and invasive mammary carcinoma. One patient had recurrent, still incompletely excised benign phyllodes tumor at the completion of this study and 131 had no evidence of disease.

Twenty-one local recurrences were observed in 18 patients (one patient with 2 recurrences and another one with 3). Thirteen phyllodes tumors recurred with the same grade ( 9 as benign with a median recurrence free interval of 30 months, 3 as borderline with a median recurrence free interval of 42 months, 1 as malignant with a medial recurrence free interval of 13 months) and 6 as more aggressive ( 2 benign recurred as borderline at a median of 34 months, 1 borderline recurred as malignant at 51 months, 3 benign recurred as malignant at a median of 111 months); for 2 recurrent malignant phyllodes tumors the histologic type of the original tumor was not known. Cases of recurrent phyllodes tumors with no follow-up data or with the original tumor not available for review were excluded from the survival analysis (all data was available for 13 phyllodes tumors that recurred locally).

Distant metastases developed exclusively in malignant tumors in $33 \%(7 / 21)$ of patients. The most prevalent metastatic site was lung $(5 / 7,71 \%)$ followed by bone, brain and pleura (2 (28\%) each) with liver, deep soft tissue, and stomach (one each); 4 patients $(57 \%)$ had metastases to 
Table 4 Features predictive of local recurrence

\begin{tabular}{|c|c|c|c|c|c|}
\hline & \multicolumn{4}{|c|}{ Univariate analysis } & \multirow{2}{*}{$\begin{array}{l}\text { Multivariate analysis } \\
P \text { value, hazard ratio }(\mathrm{HR}) \text { and } \\
\text { confidence interval }(\mathrm{CI})\end{array}$} \\
\hline & $N$ & $\begin{array}{l}\text { Local } \\
\text { recurrence }\end{array}$ & $\begin{array}{l}\text { Local } \\
\text { recurrence } \%\end{array}$ & $P$ value & \\
\hline Total & 153 & 13 & $8 \%$ & & \\
\hline Atypical fibroadenoma & 59 & 0 & 0 & 0.005 & 0.536 \\
\hline Benign phyllodes tumor & 45 & 10 & $22 \%$ & & \\
\hline Borderline phyllodes tumor & 28 & 3 & $11 \%$ & & \\
\hline Malignant phyllodes tumor & 21 & 0 & 0 & & \\
\hline Age $<50$ y & 116 & 13 & $11 \%$ & 0.026 & 0.967 \\
\hline Final margin positive & 15 & 10 & $67 \%$ & $<0.001$ & $\begin{array}{l}0.001 \\
\mathrm{HR}=16.13 \\
95 \% \text { CI }(2.9-89.6)\end{array}$ \\
\hline$\geq 50 \%$ Myxoid stroma & 59 & 9 & $15 \%$ & $<0.001$ & $\begin{array}{l}0.039 \\
\mathrm{HR}=9.63 \\
95 \% \text { CI }(1.17-77.5)\end{array}$ \\
\hline
\end{tabular}

more than one site. Axillary lymph nodes were sampled in 5 patients with malignant phyllodes tumors; two of them had metastases to axillary nodes, both developed distant metastases. All patients with distant metastases died of disease at a mean of 13 months (median of 9 months). All patients who died of disease had metastatic malignant phyllodes tumors.

Table 5 Features predictive of distant metastasis/death from disease on univariate analysis

\begin{tabular}{|c|c|c|c|c|c|c|c|}
\hline & \multirow[b]{2}{*}{$N$} & \multicolumn{3}{|c|}{$\begin{array}{l}\text { Distant metastasis-free } \\
\text { survival }\end{array}$} & \multicolumn{3}{|c|}{$\begin{array}{l}\text { Disease-specific } \\
\text { survival }\end{array}$} \\
\hline & & $\begin{array}{l}\text { Distant } \\
\text { metastasis }\end{array}$ & $\%$ & $P$ value & $\begin{array}{l}\text { Dead of } \\
\text { disease }\end{array}$ & $\%$ & $P$ value \\
\hline Total & 94 & 7 & 7 & & 7 & 7 & \\
\hline \multicolumn{8}{|c|}{ Phyllodes tumors } \\
\hline Benign & 45 & 1 & 2 & $<0.001$ & 1 & 2 & $<0.001$ \\
\hline Borderline & 28 & 0 & 0 & & 0 & 0 & \\
\hline Malignant & 21 & 6 & 29 & & 6 & 29 & \\
\hline Age $>50 y$ & 30 & 5 & 17 & 0.015 & 5 & 17 & 0.007 \\
\hline $\begin{array}{l}\text { Stromal } \\
\text { overgrowth }\end{array}$ & 29 & 6 & 21 & $<0.001$ & 6 & 21 & $<0.001$ \\
\hline $\begin{array}{l}\text { Diffuse } \\
\text { marked } \\
\text { atypia }\end{array}$ & 23 & 5 & 22 & 0.002 & 5 & 22 & 0.002 \\
\hline \multicolumn{8}{|c|}{ Mitotic index per $10 \mathrm{HPF}$} \\
\hline$<5$ & 54 & 1 & 2 & $<0.001$ & 1 & 2 & $<0.001$ \\
\hline $5-9$ & 18 & 0 & 0 & & 0 & 0 & \\
\hline$\geq 10$ & 22 & 6 & 27 & & 6 & 27 & \\
\hline Necrosis & 9 & 6 & 67 & $<0.001$ & 6 & 67 & $<0.001$ \\
\hline
\end{tabular}

\section{Features predictive of recurrence}

None of the atypical fibroadenomas recurred. The median size of phyllodes tumors that resulted in local recurrence was $3 \mathrm{~cm}$ (range: $1.7-7 \mathrm{~cm}$, mean $3.4 \mathrm{~cm}$ ). The risk of local recurrence was significantly higher in women $<50$ years old (univariate $\log$ rank test, $p=0.026$ ), tumors with positive final margin $(p<0.001$, Fig. 2a) and fibroepithelial lesions with predominantly myxoid stroma $(p<0.001)$. On multivariate analysis using Cox proportion hazard ratio, margin status and predominantly myxoid stroma were independent significant predictors for local recurrence (margin status: hazard ratio $\mathrm{HR}=16.13,95 \%$ CI 2.9-89.6, $p=0.001$; predominant myxoid stroma: $\mathrm{HR}=9.63,95 \%$ CI $1.1-7.5$, $p=0.039$, Table 4).

Tumor size, tumor border, presence of satellite/bulging nodules, stromal overgrowth, pseudoangiomatous stromal hyperplasia, stromal cellularity, diffuse marked atypia, mitotic index (regardless of counting method), adjuvant radiotherapy or personal history of fibroadenoma were not predictive of local recurrence on univariate log rank test.

The median size of malignant phyllodes that resulted in distant metastases was $10.7 \mathrm{~cm}$ (mean $10.3 \mathrm{~cm}$, range $2.7-18 \mathrm{~cm}$ ). Tumor necrosis was present in all of them. The following features were predictive of distant metastases on univariate $\log$ rank analysis: age $>50$, stromal overgrowth, diffuse marked atypia, necrosis and high mitotic index (at least 10 per $10 \mathrm{HPF}$, Table 5). However, multivariate analysis failed to identify any significant independent predictors for distant metastasis free survival $(p>0.05)$.

Tumor size, margin status, tumor border and predominantly myxoid stroma were not predictive of distant 
metastases/death from disease on univariate log rank analysis.

Pairwise comparison using Log rank test was performed comparing 4 different types of tumor border: pushing smooth (group 1), pushing irregular/minimally infiltrative (group 2), focally infiltrative (group 3) and widely infiltrative (group 4). For local recurrence free survival there was a near significant difference between group 4 and the remaining 3 groups ( $p=0.079$, Fig. 2b), while for distant metastasis free survival there was a significant difference between groups 1 and 3 ( $p=0.006)$ as well as 1 and $4(p=$ $0.029)$, and no difference between 1 and $2(\mathrm{p}=0.36)$. Similarly we compared fibroepithelial lesions in relation to their border including the presence of satellite/bulging nodules (1-pushing without nodules, 2-pushing with nodules, 3-infiltrative without nodules, 4-infiltrative with nodules); there was a significant difference between groups 1 and $3(p=0.001)$, and between groups 2 and 3 ( $p=$ $0.003)$, and a near significant difference between groups 3 and $4(p=0.074)$ for distant metastasis free survival. For local metastasis free survival there was a near significant difference between groups 1 and 4 ( $p=0.061$, Fig. 2c).

\section{Discussion}

The diagnosis of fibroepithelial lesions is frequently challenging, and the definition of pathologic criteria for their classification is a topic of ongoing debate. The lack of uniformity in definitions found in the literature certainly adds to the difficulty in reaching an evidence-based consensus. In this regard, our study offers a comprehensive morphologic analysis based on predefined criteria and long follow-up.

Most authors agree that margin status is a significant factor predictive of local recurrence $[1,2,4,9,10]$. There are however different definitions of a positive margin: "ink on tumor" vs. $<0.1 \mathrm{~mm}$ vs. $<1 \mathrm{~mm}[2,5]$. Even with an approach that only "ink on tumor" constitutes a positive margin there are cases with equivocal margin status. Fibroepithelial lesions, especially at the low end of the spectrum, usually have a well-defined, pushing border $(85 \%$ in this study), sometimes with partial or circumferential "pseudocapsule" (30\% in this study). The lesion surface can become damaged either by the surgeon while attempting a narrow excision (enucleation) to achieve the best cosmetic result or during grossing in pathology lab. This can result in the presence of ink on tumor which for shelled out lesions does not necessarily equate with a true positive margin. In our study none of the 43 fibroepithelial lesions that underwent margin re-excision for the so called "positive margin" had any residual lesion in the re-excision specimen (on rereview most of them were shelled out with rare cases having ink on tumor likely due to handling by the surgeon or during grossing). Moreover, in our study none of the 63 shelled out fibroepithelial lesions resulted in recurrence. The results of our study suggest that atypical fibroadenoma with ink on tumor do not require re-excision and, similarly to other authors $[2,5,6]$, that narrow excision appears to be an adequate treatment for benign phyllodes tumors.

There is very limited evidence in literature regarding the significance of myxoid change in fibroepithelial lesions. Frequent presence of myxoid stromal change has been noted in few, mostly remote, prior studies [10-13]. However, to our knowledge this is the first study that examined the extent of myxoid change in detail. Based on our observations it appears that phyllodes tumors with predominantly myxoid stroma may be more prone to local recurrence; this feature along with positive margin status (transected, as defined in this study) was an independent significant predictor for local recurrence on multivariate analysis using Cox proportion hazard ratio $(\mathrm{HR}=9.63$, 95\% CI 1.1-7.5, $p=0.039)$. The proportion of myxoid stroma was comparable across the entire spectrum of fibroepithelial lesions and not related to age at presentation.

In one of the more elegant studies of phyllodes tumors to date group led by I. Ellis proposed that, in order to meet the diagnostic criteria of malignant phyllodes tumor, all of the following characteristics should be present: infiltrative border in at least $50 \%$, stromal overgrowth $(4 \times)$, either marked stromal cellularity or marked pleomorphism and mitotic index of at least 10 per $10 \mathrm{HPF}$ [8]. The results of our study are in full agreement as to all but the first feature. Infiltrative border was not seen in over $40 \%$ of malignant phyllodes tumors and when present was often only focal. Therefore our study suggests that in addition to the presence of malignant heterologous element, any single one of the following features is potentially diagnostic of malignant phyllodes tumor: tumor necrosis, at least a single slide (at least $2 \times 2 \mathrm{~cm}^{2}$ ) of pure stroma, and mitotic index of $\geq 25$ per $10 \mathrm{HPF}$. On the other hand the presence of pseudoangiomatous stromal hyperplasia makes a diagnosis of malignant phyllodes tumor unlikely, which is in agreement with prior reports on this feature being more common in the benign end of the spectrum [10].

Most fibroepithelial lesions have lobular architecture (65\% in this study). These lobules or nodules can be seen in the vicinity of the main lesion (either they become detached from the main lesion or develop independently) as "satellite nodules" (34\% in this study) or be still attached and bulging out from the main lesion (15\% in this study). Whether the nodule is still attached and bulging out or already detached is often a matter of tissue orientation or plane of section. This phenomenon has been previously described by Azzopardi as "surface projections" that can contribute to tumor recurrence after enucleation [14]. In a study from MSKCC 
"fibroproliferation in surrounding tissue" was found to be predictive of local recurrence [9]. In our study the presence of satellite/bulging nodules was a significant predictor of positive final margin status $(p=0.002)$. The difference in significance could be explained by different definition and longer follow-up for the MSKCC group.

Homogeneity is uncommon among phyllodes tumors (16\% in this study). Areas resembling fibroadenoma were identified in most phyllodes (68\% in this study) and foci of hyalinized fibroadenoma, akin to the concept of carcinoma arising from pleomorphic adenoma in salivary glands, where within a carcinoma there is a hyalinized remnant of preexisting pleomorphic adenoma, were seen in $32 \%$ of phyllodes tumors. In fact the number of phyllodes tumors with a preexisting fibroadenoma may be even greater as its presence relies heavily on the thoroughness of sampling, especially in large tumors. Whether these represent remnants of preexisting fibroadenoma [15] or an intrinsic quality of phyllodes tumor [16] is still an open question. The concept that at least some phyllodes arise from fibroadenomas is supported by molecular analyses demonstrating clonal relationship and progressive alterations [17-19].

Although mitotic index by itself should not be used for classifying fibroepithelial lesions, the weight put on it differs among pathologists $([16,20]$, personal experience of the authors). The definition of the "mitotic activity" as a histologic feature seems straightforward (10 consecutive HPF in the most mitotically active area). However, the size of the field diameter, the dedication put into finding the most mitotically active area and the method one uses to select those 10 consecutive fields once in the proper area may differ. We noticed that the most mitotically active area is often only focal, confined to a single set of $10 \mathrm{HPF}$, especially in the low end of the spectrum. Increased mitotic index in fibroepithelial lesions has been described in pediatric and adolescent populations [21, 22]. We also found that patients with fibroadenomas and benign phyllodes that had mitotic index outside of the expected range tended to be younger (the mean and median age of 30 years). In our study increased mitotic index by itself was not associated with local recurrence rate either over the entire spectrum or within the low end of the spectrum alone regardless of whether one used the originally reported values or the highest counts from the most mitotically active areas. While it was not the subject of this study, there are reports on using immunohistochemistry as a surrogate marker of mitotic index in fibroepithelial tumors that might offer more objective assessment of mitotic rate [23, 24].

Our study has several limitations. This is a retrospective study with a limited sample size, issue that often exists in phyllodes tumors studies due to their rarity. Consequently variations exist with regards to grossing, extent of sampling, imaging and clinical data. While all the cases were identified from the pathology information system, some cases originated from referred consultation material where the surgery or other clinical management were performed in three additional community hospitals. A substantial number of cases included in this study were atypical fibroadenomas; however, many if not most of them would have been classified as benign phyllodes tumors by others.

In conclusion, we present a detailed morphological analysis of 213 fibroepithelial lesions that addresses features not previously reported including the frequency of fibroadenoma-like areas in phyllodes tumors, the extent of myxoid change in the stroma and the difference (or lack thereof) of using different methods of assessing mitotic index. Based on the results from this study we suggest that the presence of satellite or bulging nodules and predominantly myxoid stroma may influence the margin status and/or result in local recurrence. Increased mitotic index outside of the range from the World Health Organization guideline should be treated with caution if other worrisome features are lacking as it can be seen in indolent lesions, especially if it is only focally present after an excessively diligent search. Our study also suggests that, apart from malignant heterologous elements, the following features by itself are diagnostic of malignant phyllodes tumor: unequivocal tumor necrosis, at least a single slide $(2 \times 2$ $\mathrm{cm}^{2}$ ) of pure stroma and mitotic index of $\geq 25$ per $10 \mathrm{HPF}$. Testing on a larger cohort is necessary.

\section{CCO disclaimer}

Parts of this material are based on data and information provided by Cancer Care Ontario. However, the analysis, conclusions, opinions and statements expressed herein are those of the authors and not necessarily those of Cancer Care Ontario.

Acknowledgements We thank Dr. Gaiane Iakovleva for her help with obtaining follow-up information for few patients.

\section{Compliance with ethical standards}

Conflict of interest The authors declare that they have no conflict of interest.

\section{References}

1. Tan PH, Tse G, Lee A, Simpson JF, Hanby AM. Fibroepithelial Tumours. In: Lakhani SR, Ellis IO, Schnitt SJ, Tan PH, van de Vijver MJ editors. World Health Organization Classification of Tumours of the Breast. Lyon: IARC Press, 2012. p. 142-7.

2. Tan BY, Acs G, Apple SK, Badve S, Bleiweiss IJ, Brogi E, Calvo JP, Dabbs DJ, Ellis IO, Eusebi V, Farshid G, Fox SB, Ichihara S, 
Lakhani SR, Rakha EA, Reis-Filho JS, Richardson AL, Sahin A, Schmitt FC, Schnitt SJ, Siziopikou KP, Soares FA, Tse GM, Vincent-Salomon A, Tan PH. Phyllodes tumours of the breast: a consensus review. Histopathology. 2016;68:5-21.

3. Lawton TJ, Acs G, Argani P, Farshid G, Gilcrease M, Goldstein N, Koerner F, Rowe JJ, Sanders M, Shah SS, Reynolds. Interobserver variability by pathologists in the distinction between cellular fibroadenomas and phyllodes tumors. Int J Surg Pathol. 2014;22:695-8.

4. Tan PH, Thike AA, Tan WJ, et al. Predicting clinical behavior of breast phyllodes tumours: a nomogram based on histological criteria and surgical margins. J Clin Pathol. 2012;65:69-76.

5. Cowan ML, Argani P, Cimino-Mathews A. Benign and low-grade fibroepithelial neoplasms of the breast have low recurrence rate after positive surgical margins. Mod Pathol. 2016;29:259-65.

6. Shaaban M, Barthelmes L. Benign phyllodes tumours of the breast: (Over) treatment of margins-a literature review. Eur J Surg Oncol. 2017;43:1186-1190.

7. National Comprehensive Cancer Network . https://www.nccn.org/ professionals/physician_gls/pdf/breast.pdf. Accessed Aug 28 2017.

8. Moffat CJ, Pinder SE, Dixon AR, Elston CW, Blamey RW, Ellis IO. Phyllodes tumours of the breast: a clinicopathological review of thirty-two cases. Histopathology. 1995;27:205-18.

9. Barrio AV, Clark BD, Goldberg JI, Hoque LW, Bernik SF, Flynn LW, Susnik B, Giri D, Polo K, Patil S, Van Zee KJ. Clinicopathologic features and long-term outcomes of 293 phyllodes tumors of the breast. Ann Surg Oncol. 2007;14:2961-70.

10. Tan PH, Jayabaskar T, Chuah KL, Lee HY, Tan Y, Hilmy M, Hung H, Selvarajan S, Bay BH. Phyllodes tumors of the breast: the role of pathologic parameters. Am J Clin Pathol. 2005;123:529-40.

11. Lee BJ, Pack GT. Giant intracanalicular fibroadenomyxoma of the breast: the so-called cystosarcoma phylloides mammae of Johannes Muller. Am J Cancer. 1931;15:2583-609.

12. Norris HJ, Taylor HB. Relationship of histologic features to behavior of cystosarcoma phyllodes. Analysis of ninety-four cases. Cancer. 1967;20:2090-9.

13. Kleer CG, Giordano TJ, Braun T, Oberman HA. Pathologic, immunohistochemical, and molecular features of benign and malignant phyllodes tumors of the breast. Mod Pathol. 2001;14:185-90.

14. Azzopardi JG. Problems in Breast Pathology. WB Saunders, 1979: p. 346-365.

15. Koerner FC. Diagnostic problems in breast pathology. Saunders Elsevier, 2009. p. 329-41.

16. Brogi E. Fibroepithelial neoplasms. In Hoda SA, Brogi E, Koerner FC, Rosen PP. Rosen's Breast pathology. Wolters Kluwer, 2014. p. $213-70$.

17. Hodges KB, Abdul-Karim FW, Wang M, et al. Evidence for transformation of fibroadenoma of the breast to malignant phyllodes tumor. Appl Immunohistochem Mol Morphol. 2009; 17:345-50.

18. Kuijper A, Buerger H, Simon R, Schaefer KL, Croonen A, Boecker W, van der Wall E, van Diest PJ. Analysis of the progression of fibroepithelial tumours of the breast by PCR-based clonality assay. J Pathol. 2002;197:575-81.

19. Foucar CE, Hardy A, Siziopikou KP, et al. A mother and daughter with phyllodes tumors of the breast. Clin Breast Cancer. 2012;12:373-7.

20. Yom CK1, Han W, Kim SW, Park SY, Park IA, Noh DY. Reappraisal of conventional risk stratification for local recurrence based on clinical outcomes in 285 resected phyllodes tumors of the breast. Ann Surg Oncol. 2015;22:2912-8.

21. Tay TKY, Chang KTE, Thike AA, Tan PH. Paediatric fibroepithelial lesions revisited: pathological insights. J Clin Pathol. 2015;68:633-41.

22. Ross DS, Giri DD, Akram MM, Catalano JP, Olcese C, Van Zee KJ, Brogi E. Fibroepithelial lesions in the breast of adolescent females: a clinicopathological study of 54 cases. Breast J. 2017;23:182-92.

23. Korcheva VB, Levine J, Beadling C, Warrick A, Countryman G, Olson NR, Heinrich MC, Corless CL, Troxell ML. Immunohistochemical and molecular markers in breast phyllodes tumors. Appl Immunohistochem Mol Morphol. 2011;19:119-25.

24. Ginter PS, Shin SJ, Liu Y, Chen Z, D'Alfonso TM. Phosphohistone $\mathrm{H} 3$ expression correlates with manual mitotic counts and aids in identification of "hot spots" in fibroepithelial tumors of the breast. Hum Pathol. 2016;49:90-8. 